\title{
An Empirical Study of Code Clone Genealogies
}

\author{
Miryung Kim, Vibha Sazawal, \\ David Notkin \\ Computer Science \& Engineering \\ University of Washington \\ Seattle, Washington USA \\ \{miryung,vibha,notkin\}@cs.washington.edu
}

\author{
Gail C. Murphy \\ Department of Computer Science \\ University of British Columbia \\ Vancouver, B.C. Canada \\ murphy@cs.ubc.ca
}

\begin{abstract}
It has been broadly assumed that code clones are inherently bad and that eliminating clones by refactoring would solve the problems of code clones. To investigate the validity of this assumption, we developed a formal definition of clone evolution and built a clone genealogy tool that automatically extracts the history of code clones from a source code repository. Using our tool we extracted clone genealogy information for two Java open source projects and analyzed their evolution.

Our study contradicts some conventional wisdom about clones. In particular, refactoring may not always improve software with respect to clones for two reasons. First, many code clones exist in the system for only a short time; extensive refactoring of such short-lived clones may not be worthwhile if they are likely diverge from one another very soon. Second, many clones, especially long-lived clones that have changed consistently with other elements in the same group, are not easily refactorable due to programming language limitations. These insights show that refactoring will not help in dealing with some types of clones and open up opportunities for complementary clone maintenance tools that target these other classes of clones.
\end{abstract}

\section{Categories and Subject Descriptors}

D.2.7 [Software Engineering]: Distribution, Maintenance, and Enhancement-restructuring, reverse engineering, and reengineering

\section{General Terms}

Measurement, Experimentation

\section{Keywords}

software evolution, code clone, empirical study, software maintenance, refactoring

Permission to make digital or hard copies of all or part of this work for personal or classroom use is granted without fee provided that copies are not made or distributed for profit or commercial advantage and that copies bear this notice and the full citation on the first page. To copy otherwise, to republish, to post on servers or to redistribute to lists, requires prior specific permission and/or a fee.

ESEC-FSE'05, September 5-9, 2005, Lisbon, Portugal

Copyright 2005 ACM 1-59593-014-0/05/0009 ...\$5.00.

\section{INTRODUCTION}

The presence of code clones - code snippets that are similar in syntax and semantics - is generally considered to be an indication of poor software quality. The primary concern is that programmers may introduce bugs when changing code if they inadvertently neglect to change related clones.

This view has directed the goals of previous research efforts - finding code clones and aggressively removing them. Many efforts have focused on automatically identifying code clones $[6,10,13,18,19,22,24,26,30]$ and on using the output of clone detection as a basis for refactoring [8, 17, 23]. Some methodologists have educated programmers about how to avoid or remove code clones. For example, Fowler [14] argues that code duplicates are bad smells of poor design and programmers should aggressively use refactoring techniques. As another example, the Extreme Programming (XP) [11] community has integrated frequent refactoring as a part of the development process and has argued that fewer clones are found in XP process software [30].

We became skeptical about the universal validity of this conventional wisdom after studying copy and paste programming practices in industry [20]. In the study, we found that skilled programmers often created and managed code clones with clear intent. Subjects copied and pasted code snippets to reuse logic that could not be abstracted due to the limitations of the Java programming language. In addition, our subjects often appeared to discover a shared abstraction of similar code through the process of copying, pasting, and modifying code; they kept and maintained clones for some period of time before they realized how to abstract the common part of the clones.

Our previous study suggested that the practice of creating and managing clones is not necessarily bad and motivated us to investigate how clones evolve over time. So we performed a longitudinal analysis of how clones change over the lifetime of software. To enable this analysis, we defined a formal model of clone evolution and built a tool that automatically extracts clone genealogies - the history of how each element in a group of clones has changed with respect to other elements in the same group - from a set of program versions. Using this tool, we studied, in two Java open source projects, (1) how long clones survive in a system and (2) how often and in which way clones change.

In summary, we found the following:

- Consistent with conventional wisdom, clones impose obstacles during software evolution because they often change similarly with their counterparts in the same 
group, thus requiring programmers update the same change repeatedly. In our study, $36 \%$ to $38 \%$ of clone genealogies consist of clones that have changed consistently with other elements in the same group.

- Contrary to conventional wisdom, popular refactoring techniques [14] cannot easily remove many long-lived clones that change consistently with other clones. In the systems we studied, we found that $49 \%$ to $64 \%$ of clone genealogies consist of clones that cannot be easily removed using standard refactoring techniques. In fact, the longer code clones survive in the system, the more they represent this type of unfactorable clones that have changed consistently with other clones.

- In the systems that we studied, we found that many clones were volatile; among the clone genealogies that disappeared during evolution, $48 \%$ to $72 \%$ disappeared within an average of eight check-ins (out of over at least 160 check-ins). If clones in the same group are to change differently from one another in a short time, aggressive, immediate refactoring may not be necessary or beneficial.

The results suggest understanding the characteristics of how clones actually evolve may open new approaches to clone management.

The rest of this paper is organized as follows. Section 2 describes related work. Section 3 formally defines the model of clone evolution, which serves as the basis of our clone genealogy extractor described in Section 4. Section 5 describes our study procedure, and Section 6 presents analysis of clone evolution patterns and discusses implications of our results. Section 7 discusses our study limitations. Section 8 proposes clone maintenance tools that use clone genealogy information, and Section 9 summarizes our study.

\section{RELATED WORK}

Automatic Detection of Code Clones. Although most consider code clones to be identical or near identical fragments of source code $[9,19]$, code clones have no consistent or precise definition in the literature. Indeed, a "clone" has been defined operationally based on the computation of individual clone detectors.

Clone detectors can be grouped into four basic approaches, each of which uses a different representation of source code and different algorithms for comparing the representation of potential clones.

First, some detectors are based on lexical analysis. For instance, Baker's [6] Dup uses a lexer and a line-based string matching algorithm. Dup removes white spaces and comments; replaces identifiers of functions, variables, and types with a special parameter; concatenates all files to be analyzed into a single text file; hashes each line for comparison; and extracts a set of pairs of longest matches using a suffix tree algorithm. Kamiya et al. improved Dup's algorithm and developed CCFinder [19], which transforms tokens of a program according to a language-specific rule and performs a token-by-token comparison. CCFinder is recognized as a state of the art clone detector that handles industrial size programs; it is reported to produce higher recall although its precision is lower than some other tools [12]. CP-Miner [26] identifies a similar sequence of tokenized statements using a frequent subsequence mining technique.
Second, Baxter et al. developed CloneDr [10], which parses source code to build an abstract syntax tree and compares its subtrees by characterization metrics (hash functions).

Third, some detectors find clones by identifying an isomorphic program dependence graph. Komondoor and Horwitz's clone detector finds isomorphic PDG subgraphs using program slicing [22]. Krinke uses a $k$-length patch matching to find similar PDG subgraphs [24]. PDG-based clone detection is robust to reordered statements, insertion and deletion of code, intertwined code, and non-contiguous code, but it is not scalable to large size programs.

Finally, metric-based clone detectors [18, 27, 28] compare various software metrics called fingerprinting functions. These clone detectors find clones in a particular syntactic granularity such as a class, a function, or a method, because fingerprinting functions are often defined for a particular syntactic unit.

Reengineering the Output of a Clone Detector. Researchers have also used the output of a clone detector as a basis for refactoring. For example, Balazinska et al. developed a clone reengineering tool, called CloRT $[7,8]$. CloRT finds clones using software metrics and a dynamic pattern matching algorithm, determines whether the Strategy or Template design pattern applies to these clones, factors out the common parts of methods, and parametrizes the differences with respect to the design patterns. As another example, Komondoor and Horwitz developed a semanticspreserving procedure extraction algorithm that runs on PDGbased clones [22, 23]. Finally, CCShaper [17] filters the output of CCFinder to find good candidates for the extract method and pull up method refactoring patterns.

Studies of Clone Coverage. Several studies have investigated the extent of code clones. Comparing the result of these studies is difficult because the definition of a clone depends on the computation of individual clone detectors and many detection algorithms take adjustable parameters. Nearly as much as $10 \%$ to $30 \%$ of the code in many large scale projects was identified as code clones (e.g., gcc-8.7\% [13], JDK-29\% [19], Linux-22.7\% [26], etc). Antoniol et al. [5] and Li et al. [26] studied changes in clone coverage (the ratio of cloned code to the total lines of code) in Linux and found that clone coverage increased early in development but stabilized over time. They interpreted these data as showing that the design of Linux is not deteriorating due to copy and paste practices. These quantitative studies of clones do not address how clones change over time and whether it is difficult for developers to manage code clones.

A Study of Changes of Clones. Evolution of code clones was studied for the first time by Laguë et al. [25]. They studied clones in six versions of a large telecommunication software and found that a significant number of clones were removed but the overall number of clones increased over time in the system. Their approach traces code clones in consecutive versions using a metric-based clone detector and classifies clones into four categories: new clones, modified clones, never modified clones, and deleted clones. Their analysis does not address how elements in a group of code clones change with respect to other elements in the group. To the best of our knowledge, our clone genealogy extractor (detailed in Section 4) is the first tool that systematically analyzes clone evolution patterns by monitoring how a clone group evolves. 
Techniques for Analyzing Structural Changes. Origin analysis $[15,35]$ is similar to our genealogy analysis (described in detail in Section 3 and 4) because it employs a cloning relationship to trace code fragments across versions. The goal of origin analysis is to understand structural changes during evolution, and it has been applied to detect splitting and merging of code fragments. However it differs from our analysis that (1) it semi-automatically traces only code fragments specified by a user and (2) it does not monitor operational changes to a group of code clones, such as whether clones change consistently (or inconsistently) with other elements in the same group.

Antoniol et al. proposed an automatic approach, based on vector space information retrieval, to identify several refactoring events, namely class renaming, replacement, merge, and split [4]. These analyses do not focus on structural changes of code clones.

\section{MODEL OF CLONE GENEALOGY}

To study clone evolution structurally and semantically rather than quantitatively, we defined a model of clone genealogy. The genealogy of code clones describes how groups of code clones change over multiple versions of a program. In a clone's genealogy, a group to which the clone belongs is traced to its origin clone group in the previous version. The model associates related clone groups that have originated from the same ancestor clone group. In addition, the genealogy contains information about how each element in a group of clones has changed with respect to other elements in the same group. We wrote our model in the Alloy modeling language [3] to check whether several evolution patterns can describe all possible changes to a clone group and to clarify the relationship among evolution patterns. (Our entire model is available on the web [1].)

The basic unit in our model is a Code Snippet, which has two attributes, Text and Location. Text is an internal representation of code that a clone detector uses to compare code snippets. For example, when using CCFinder [19], text is a parametrized token sequence, whereas when using Clone Dr [10], text is an isomorphic AST. A Location is used to trace code snippets across multiple versions of a program; thus, every code snippet in a particular version of a program has a unique location. To determine how much the text of a code snippet has changed across versions, we define a TextSimilarity function that measures the text similarity between two texts $t 1$ and $t 2(0 \leq$ TextSimilarity $(t 1, t 2) \leq 1)$. To trace a code snippet across versions, we define a LocationOverlapping function that measures how much two locations $l 1$ and $l 2$ overlap each other $(0 \leq$ LocationOverlapping $(l 1, l 2) \leq 1)$. A Clone Group is a set of code snippets with identical text. $C G$.text is a syntactic sugar for the text of any code snippet in a clone group $C G$. A Cloning Relationship is defined between two clone groups $C G_{1}$ and $C G_{2}$ if and only if TextSimilarity $\left(C G_{1}\right.$.text, $C G_{2}$.text $) \geq \operatorname{sim}_{t h}$, where $\operatorname{sim}_{t h}$ is a constant between 0 and 1. An Evolution Pattern is defined between an old clone group $O G$ in the $k-1^{t h}$ version and a new clone group $N G$ in the $k^{t h}$ version such that there exists a cloning relationship between $N G$ and $O G$.

We defined several evolution patterns that describe all possible changes to a clone group. The patterns are defined for clone groups instead of individual clone snippets because we are interested in understanding whether programmers update (or forget to update) a group of clones similarly.

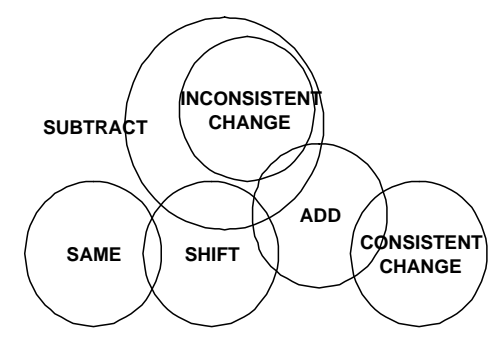

Figure 1: The relationship among evolution patterns

- Same: all code snippets in $N G$ did not change from $O G$.

TextSimilarity (NG.text,OG.text) $=1$

all cn:CodeSnippet | some co:CodeSnippet $\mid \mathrm{cn}$ in NG $\Rightarrow$ co in OG \&\& LocationOverlapping $(\mathrm{cn}, \mathrm{co})=1$

all co:CodeSnippet | some cn:CodeSnippet | co in OG $\Rightarrow$ $\mathrm{cn}$ in NG \&\& LocationOverlapping $(\mathrm{cn}, \mathrm{co})=1$

- Add: at least one code snippet in $N G$ is newly added. For example, programmers added a new code snippet to $N G$ by copying an old code snippet in $O G$.

TextSimilarity(NG.text,OG.text) $\geq \operatorname{sim}_{t h}$

some cn:CodeSnippet | all co:CodeSnippet | co in OG $\Rightarrow$ cn in NG \&\& LocationOverlapping $(\mathrm{cn}, \mathrm{co})=0$

- Subtract: at least one code snippet in $O G$ does not appear in $N G$. For example, programmers refactored or removed a code clone.

TextSimilarity(NG.text,OG.text) $\geq \operatorname{sim}_{t h}$ some co:CodeSnippet | all cn:CodeSnippet | cn in NG $\Rightarrow$ $\mathrm{co}$ in OG \&\& LocationOverlapping $(\mathrm{cn}, \mathrm{co})=0$

- Consistent Change: all code snippets in $O G$ have changed consistently; thus they belong to $N G$ together. For example, programmers applied the same change consistently to all code clones in $O G$.

$\operatorname{sim}_{t h} \leq$ TextSimilarity(NG.text,OG.text $)<1$ all co:CodeSnippet | some cn:CodeSnippet $\mid$ co in OG $\Rightarrow$ $\mathrm{cn}$ in NG \&\& LocationOverlapping $(\mathrm{cn}, \mathrm{co})>0$

- Inconsistent Change: at least one code snippet in $O G$ changed inconsistently; thus it does not belong to $N G$ anymore. For example, a programmer forgot to change one code snippet in $O G$.

$\operatorname{sim}_{t h} \leq$ TextSimilarity(NG.text,OG.text) $<1$

some co:CodeSnippet | all cn:CodeSnippet $\mid \mathrm{cn}$ in NG $\Rightarrow$ co in OG \&\& LocationOverlapping $(\mathrm{cn}, \mathrm{co})=0$

- Shift: at least one code snippet in $N G$ partially overlaps with at least one code snippet in $O G$.

TextSimilarity(NG.text,OG.text) $=1$

some cn:CodeSnippet | some co:CodeSnippet | cn in NG \&\& co in OG \&\& ( $1>$ LocationOverlapping $(\mathrm{cn}, \mathrm{co})>0)$

In our model, different kinds of evolution patterns may overlap. For example, a change to a clone group can represent both the Consistent Change pattern and the Add pattern. We used the Alloy analyzer to check whether the combination of patterns can describe all possible changes to a clone group. In our initial attempt, we discovered the Shift pattern, which is a somewhat unintuitive but necessary pattern 


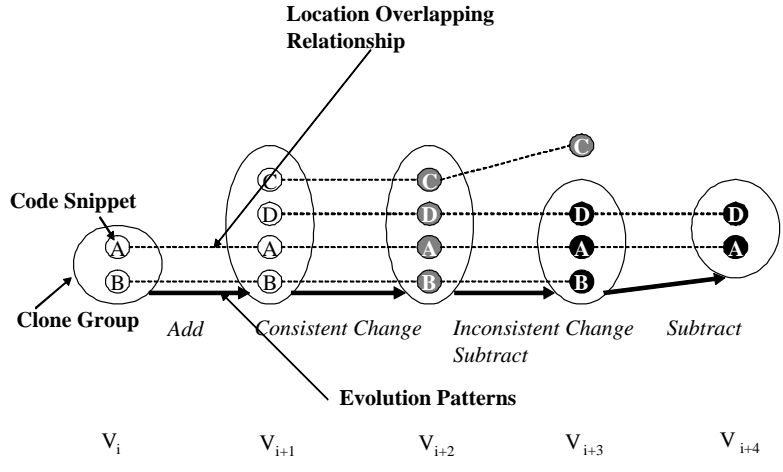

Figure 2: An example clone lineage

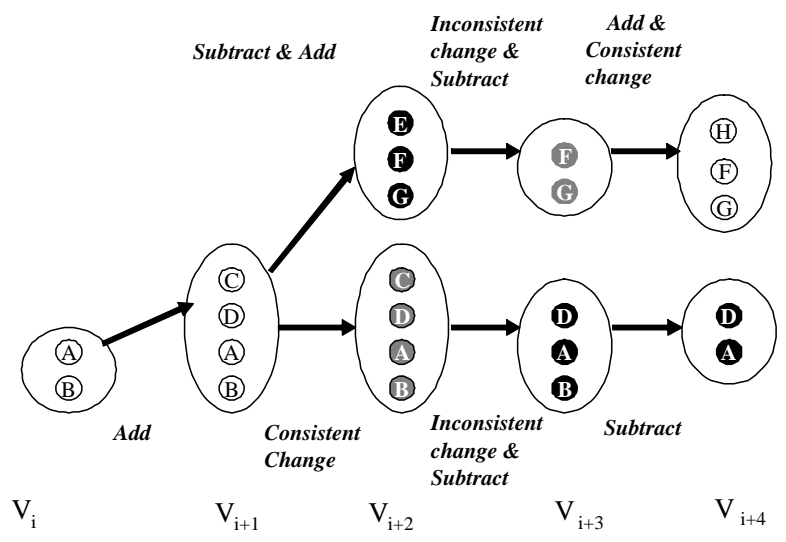

Figure 3: An example clone genealogy

to cover all possible changes to a clone group. The relationship among evolution patterns is validated by the Alloy analyzer and is described in the Venn diagram in Figure 1.

A Clone Lineage is a directed acyclic graph that describes the evolution history of a sink node (clone group). In a clone lineage, a clone group in the $k^{t h}$ version is connected by an evolution pattern from a clone group in the $k-1^{t h}$ version. For example, Figure 2 shows a clone lineage including the Add, Consistent Change, Inconsistent Change, and Subtract patterns. In the figure, code snippets with the same text are filled with the same color.

A Clone Genealogy is a set of clone lineages that have originated from the same clone group. A clone genealogy is a connected component where every clone group is connected by at least one evolution pattern. ${ }^{1}$ A clone genealogy approximates how programmers create, propagate, and evolve code clones. For example, Figure 3 shows a clone genealogy that comprises two clone lineages.

\section{CLONE GENEALOGY EXTRACTOR}

Based on the model in Section 3, we built a tool that auto-

\footnotetext{
${ }^{1} \mathrm{~A}$ clone genealogy is a connected component in the sense that there exists an undirected path for every pair of clone groups. Although a clone genealogy is often an inverted tree in practice, it is a connected component in theory because the in-degree of a new clone group can be greater than one when it is ambiguous to determine the most likely origin of a new clone group.
}

matically extracts clone genealogies over a project's lifetime. Our clone genealogy extractor (CGE) requires three inputs: (1) multiple versions of a program in a chronological order, $\left\{V_{k} \mid 1 \leq k \leq n\right\}$, (2) a clone detector, and (3) a location tracker that traces a code snippet's location across versions.

To assist a user of CGE to prepare multiple versions of a program, CGE automatically extracts all versions of a project in a chronological order from its source code repository (CVS). Because CVS records individual file revisions but not which files were changed together, CGE uses Kenyon's [16] front-end to identify CVS check-in transactions and to check out the source code that corresponds to each check-in. Depending on the granularity of evolution analysis, a user can select a subset of versions. For example, a user can select all versions corresponding to all check-ins or only the versions that increased (or decreased) the total number of lines of code clones (LOCC).

CGE identifies clone groups in each version $V_{k}$ using a clone detector. Currently we use CCFinder [19] described in Section 2, but any clone detector can be used.

CGE also implements the TextSimilarity function using a clone detector. CGE currently identifies the common part between two texts $t 1$ and $t 2$ using CCFinder and calculates the common part's relative proportion to the size of $t 1$ and $t 2$.

$$
\text { TextSimilarity }(t 1, t 2)=\frac{2|t 1 \bigcap t 2|}{|t 1|+|t 2|}
$$

where $|t|$ is the size of text $t$ and $t 1 \bigcap t 2$ is the common part of $t 1$ and $t 2$. Using a different type of clone detector may require reimplementing the TextSimilarity function.

CGE uses a location tracker to implement the LocationOverlapping function, which computes an overlapping score between a location $L_{i}$ in $V_{k-1}$ and a location $L_{j}$ in $V_{k}$. CGE currently uses a file and line based location tracker that we built on top of diff. The diff-based location tracker maps the line numbers of $L_{j}$ to old line numbers in the same file $f$ in $V_{k-1}$ based on the differences caused by insertion or deletion of code. Then it computes the relative proportion of an overlapped region between $L_{i}$ and the calibrated $L_{j}$.

LocationOverlapping $\left(L_{i}, L_{j}\right)=\frac{\min \left(n_{e}, o_{e}\right)-\max \left(n_{s}, o_{s}\right)}{n_{e}-n_{s}}$

where $L_{i}$ spans from the line $o_{s}$ to the line $o_{e}$, and the calibrated location of $L_{j}$ in $V_{k-1}$ spans from the line $n_{s}$ to the line $n_{e}$. Using a different type of location tracker may require reimplementing the LocationOverlapping function.

Using the same clone detector, CGE finds cloning relationships between the clone groups in $V_{k-1}$ and the clone groups in $V_{k}$ for $1<k \leq n$. Given a clone group in $V_{k}$, a clone detector may find several cloning relationships to clone groups in $V_{k-1}$. CGE applies a heuristic to determine the most likely origin (a clone group in $V_{k-1}$ ) and to remove less interesting cloning relationships. Our heuristic first selects a cloning relationship with the best location overlapping score. If a cloning relationship with the best similarity score is different from the one with the best location overlapping score, our heuristic selects both because of ambiguity.

After applying the heuristic, CGE separates each connected component of cloning relationships. Then, it labels evolution patterns in each connected component, a clone genealogy. CGE visualizes a genealogy graph using the Graphviz package [2] and allows a user to browse code relevant to a selected genealogy. 


\section{STUDY PROCEDURE}

Our CGE captures various kinds of clone evolution patterns and thus allows us to explore a wide variety of research questions about clone evolution. In this study, we focused on the following questions: (1) how often do programmers update clones consistently? (2) how long do clones live in the system? and (3) what are evolutionary characteristics of clones that cannot be easily removed with refactoring techniques?

To determine these characteristics, we chose two subject programs with a significant evolutionary history and applied our tool to extract clone genealogies from those programs. Because the clone detector can introduce false positives, we manually removed some of the extracted genealogies. We then analyzed the remaining genealogies and computed the age of the genealogies and the kinds of evolution patterns they include.

Since our previous copy and paste study [20] had focused on Java programs, we decided to focus on subject programs written in Java. We selected carol and dnsjava because they met this condition and both had CVS history for over two years. In addition, their code size allowed us to manually inspect genealogies if necessary. Carol is a library that allows clients to use different RMI (remote method invocation) implementations and has evolved over 26 months from August 2002 to October 2004. Dnsjava is an implementation of DNS (domain name system) in Java that has evolved over 74 months from September 1998 to November 2004. Table 1 describes the programs' size in lines of code (LOC), the period that we studied, and the number of CVS check-ins during the period. ${ }^{2}$ We studied the history of dnsjava from March 1999 (the first release) because many directories were duplicated for file back up and they were not cleaned up until the first release.

For our analysis, we focused on versions of the programs in which the LOCC (the total number of lines of code clones) increased or decreased from the preceding version; this approximates the set of program versions that added or deleted code clones or made changes to code clones. For our target programs, this resulted in studying 37 versions out of 164 versions of carol and 224 versions out of 905 versions of $d n s$ java.

We used 30 tokens (a default setting) as a minimum token length for CCFinder because programmers do not generally consider very short code snippets as code clones, and many research projects have used this default setting to find code clones. Setting the minimum at 30 tokens resulted in an average clone size of seven lines in carol and dnsjava. With this setting, CCFinder found average clone coverage ratio of $10.6 \%$ in carol and $10.5 \%$ in dnsjava.

We set the threshold sim $_{t h}$ of TextSimilarity function to be 0.3 because we empirically found that 0.3 neither underestimates nor overestimates the size and the length of genealogies. We discuss how $\operatorname{sim}_{t h}$ affects our results in detail in Section 7.1.

CCFinder occasionally detects false positive clones that are similar in a token sequence, although common sense says that they are not clones. We used our previously defined concept of a "syntactic template" to identify clones as false

\footnotetext{
${ }^{2} \mathrm{~A}$ check-in in our analysis corresponds to a single logical CVS transaction that commits a set of revisions together within a time window of 3 minutes [34].
}

Table 1: Description of Two Java Subject Programs

\begin{tabular}{|c||c|c|}
\hline Program & carol & dnsjava \\
\hline URL & carol.objectweb.org & www.dnsjava.org \\
\hline LOC & $7878 \sim 23731$ & $5756 \sim 21188$ \\
\hline duration & 26 months & 68 months \\
\hline \# of check-ins & 164 & 905 \\
\hline
\end{tabular}

Table 2: Clone Genealogies in carol and dnsjava $\left(\right.$ min $_{\text {token }}=\mathbf{3 0}$, sim $\left._{\text {th }}=0.3\right)$

\begin{tabular}{|c||c|c|}
\hline \# of genealogies & carol & dnsjava \\
\hline total & 122 & 140 \\
\hline false positive & 13 & 15 \\
\hline true positive & 109 & 125 \\
\hline locally unfactorable & $70(64 \%)$ & $61(49 \%)$ \\
\hline consistently changed & $41(38 \%)$ & $45(36 \%)$ \\
\hline
\end{tabular}

positives. The idea of a syntactic template comes from our study of copy and paste programming [20]. A syntactic template is a template of repeated code appearing in a row because a programmer often copies and pastes a code fragment when writing a series of syntactically similar code fragments. For example, a programmer often copies a field declaration statement when writing a block of field declaration, an invocation statement when writing a static initializer, or a case statement when writing a series of case statements in a switch-case block. We manually removed 13 out of 122 genealogies in carol and 15 out of 140 genealogies in dnsjava because they consist of only syntactic templates (see Table 2). Although there could be false negative clones that CCFinder cannot find, we do not think that there are many because a previous comparison of clone detectors [12] suggests that CCFinder has a much higher recall than CloneDr (AST-based) [10] or Covet (metric-based) [27], although its precision is lower than the CloneDr.

\section{STUDY RESULTS}

This section presents the evolution patterns of code clones in carol and dnsjava and answers the questions raised in Section 5 .

\subsection{Consistently Changing Clones}

To determine how often code clones change consistently with other clones in the same clone group, we measured the number of genealogies with a consistent change pattern. Throughout this paper, we use a genealogy instead of a lineage as our measurement unit because (1) lineages in the same genealogy stem from the same clone group, thus inheriting the same evolution patterns and (2) a clone group's location in one lineage may overlap with that of other lineages in the same genealogy.

We say that a clone genealogy includes a consistently changing pattern if and only if all lineages in the clone genealogy include at least one "consistent change" pattern.

Out of 109 genealogies in carol, 41 genealogies (38\%) include a consistently changing pattern. Out of 125 genealogies in dnsjava, 45 genealogies (36\%) include a consistently changing pattern. So, consistent with conventional wisdom, many of the clones in the study impose the challenge of consistent update on programmers. 

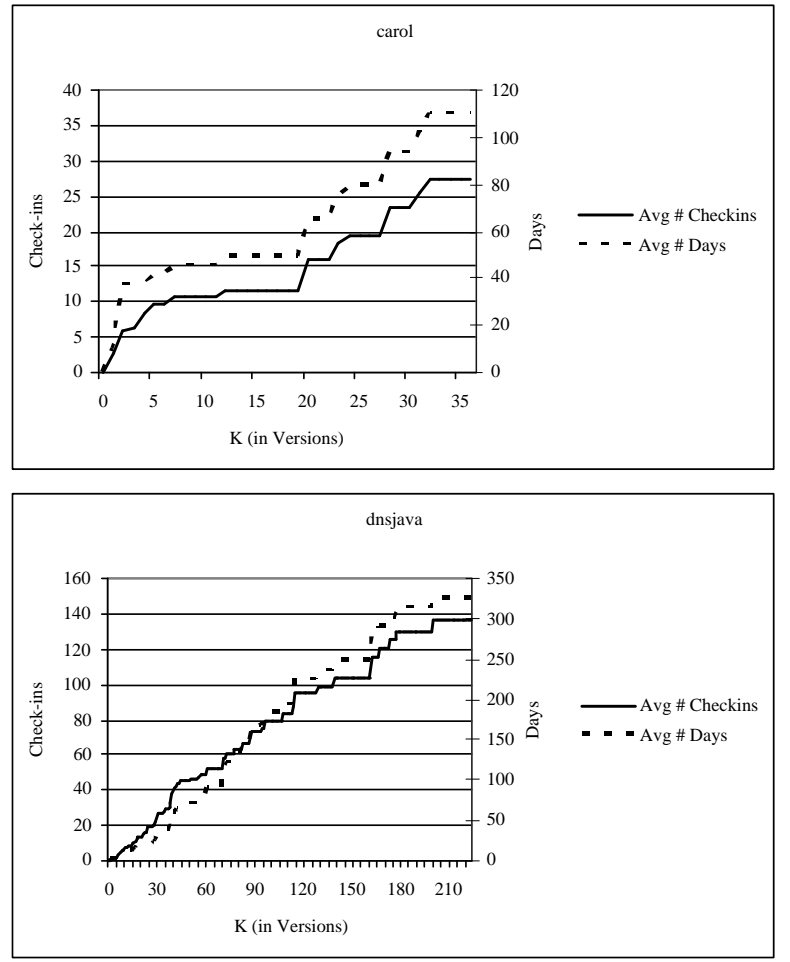

Figure 4: The average lifetime of $k$-volatile clone genealogies

\subsection{Volatile Clones}

To understand how long clones survive in the systems, we measured the age of a clone genealogy - how many versions (generations) a genealogy spans. In our analysis, we classified genealogies in two groups, dead genealogies that do not include clone groups of the final version and alive genealogies that include clone groups of the final version. We differentiate a dead genealogy from an alive genealogy because the age of a dead genealogy provides information about how long clones stayed in the system before they disappeared. On the other hand, for an alive genealogy, we cannot tell how long its clones will survive because they are still evolving. At the end point of our analysis, in carol, out of 109 clone genealogies, 53 of them are dead and 56 of them are alive. In dnsjava, out of 125 clone genealogies, 107 of them are dead and 18 of them are alive.

To reason about how long genealogies survived in terms of absolute time as well as in the number of versions used in our analysis, we define $k$-volatile genealogies and measure the average lifetime of $k$-volatile genealogies. $k$-volatile genealogies are clone genealogies that have disappeared within $k$ versions, i.e., $k$-volatile genealogies $=\{g \mid g$ is a dead genealogy and $0 \leq g$.age $\leq k\}$. Figure 4 shows the average lifetime of $k$-volatile genealogies in the number of check-ins (left axis) and the number of days (right axis). Let $f(k)$ be the number of genealogies with the age $k$ and $f_{\text {dead }}(k)$ be the number of dead genealogies with the age $k . C D F_{\text {dead }}(k)$ is a cumulative distribution function of $f_{\text {dead }}(k)$ and it means the ratio of $k$-volatile genealogies among all dead genealogies. Let $R_{\text {volatile }}(k)$ be the ratio of $k$-volatile genealogies
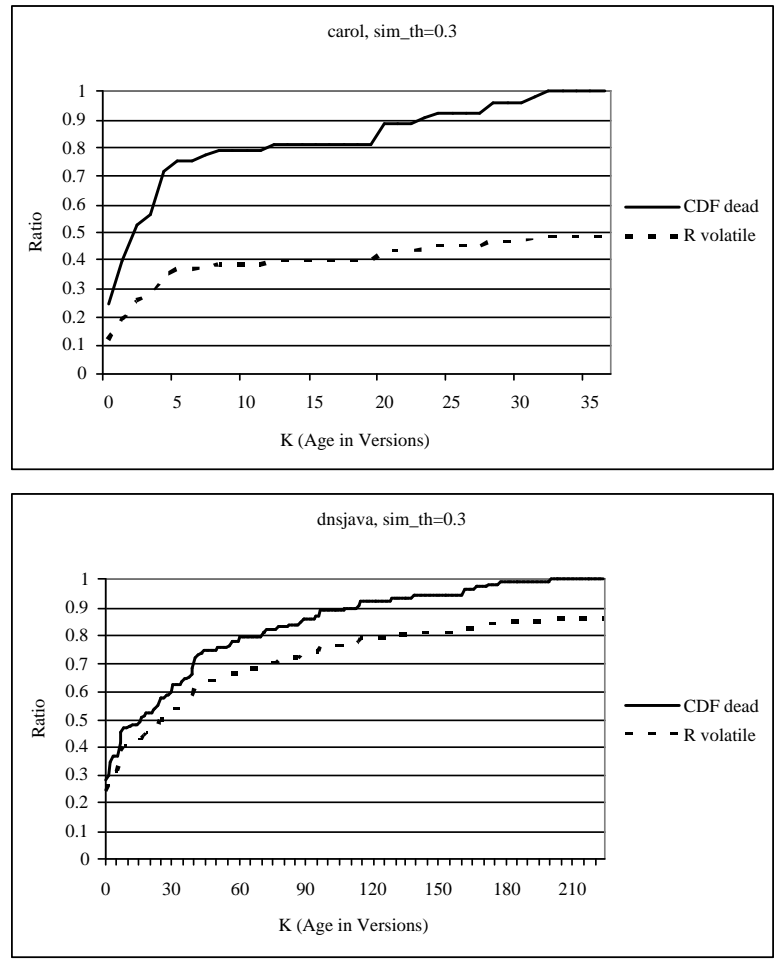

Figure 5: $C D F_{\text {dead }}(k)$ and $R_{\text {volatile }}(k)$ of carol and $\boldsymbol{d n s}$ java

among all genealogies in the system.

$$
\begin{gathered}
C D F_{\text {dead }}(k)=\frac{\sum_{i=0}^{k} f_{\text {dead }}(i)}{\sum_{i=0}^{n} f_{\text {dead }}(i)} \\
R_{\text {volatile }}(k)=\frac{\sum_{i=0}^{k} f_{\text {dead }}(i)}{\sum_{i=0}^{n} f(i)}
\end{gathered}
$$

Figure 5 presents $C D F_{\text {dead }}(k)$ and $R_{\text {volatile }}(k)$ for carol and dnsjava. In carol, $37 \%$ of all genealogies ( $75 \%$ of dead genealogies) have disappeared within 5 versions, and $39 \%$ of all genealogies (79\% of dead genealogies) have disappeared within 10 versions. When we interpret these data in the number of check-ins or in the number of days by referring to Figure 4, they mean that $37 \%$ of all genealogies lasted an average of 9.6 check-ins and 41.7 days and $39 \%$ lasted an average of 10.8 checkins and 45.6 days during the evolution period of 164 check-ins and 800 days in carol.

In dnsjava, $31 \%$ of all genealogies (36\% of dead genealogies) have disappeared within 5 versions, and $41 \%$ of all genealogies ( $48 \%$ of dead genealogies) have disappeared within 10 versions. These data mean that $31 \%$ of all genealogies lasted an average of 1.48 check-ins and 1.48 days and $41 \%$ lasted an average of 7.35 check-ins and 11.05 days during the evolution period of 905 check-ins and 2051 days in dnsjava.

So in both systems, a large number of clones were volatile. The large extent of volatile clones suggests that a substantial amount of the work done by a developer applying a strategy of aggressive, immediate refactoring may not be cost-effective. When we manually inspected all dead lineages, we found that $26 \%$ (carol) to $34 \%$ (dnsjava) of them were discontinued because of divergent changes in the clone 

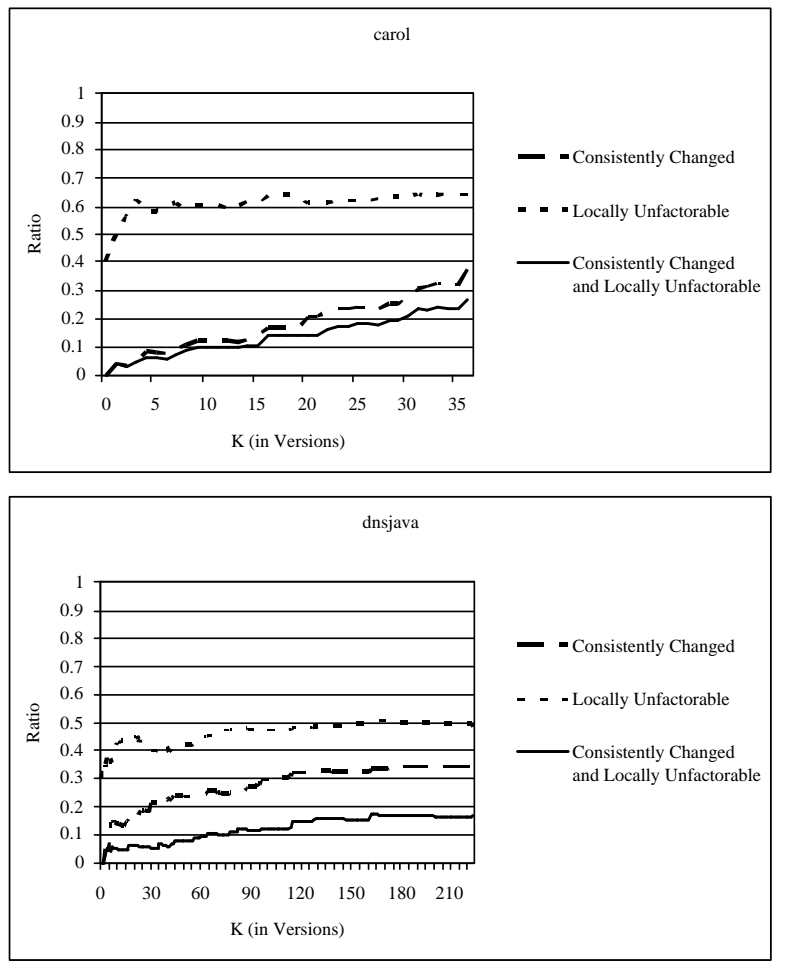

Figure 6: Cumulative fraction of consistently changed genealogies, locally unfactorable genealogies, and consistently changed and locally unfactorable genealogies

\section{DISCUSSION}

Section 7.1 discusses how the text similarity threshold affects our analysis results and Section 7.2 describes limitations of our study.

\subsection{Similarity Threshold}

The text similarity threshold, $\operatorname{sim}_{t h}$, sets the bar for defining a cloning relationship, so it could affect the size and length (age) of clone genealogies. Ultimately $\operatorname{sim}_{t h}$ could affect the number of volatile clones and consistently changing clones because a low sim $\sin _{\text {w }}$ would find a consistent change pattern between OG and NG while a high $\operatorname{sim}_{t h}$ would consider that OG's lineage is discontinued.

Table 4 shows that, when 0.1 is used, CGE finds fewer genealogies with a larger size and a longer length because it finds more cloning relationships and thus combines several genealogies to one. When 0.5 is used, CGE finds more genealogies with a smaller size and a shorter length because it breaks a long clone genealogy into many short and small genealogies. When $\operatorname{sim}_{t h}$ is 0.1 , the ratio of consistently changed genealogies is $2 \%$ higher in dnsjava and $26 \%$ higher in carol than using 0.3. Figure 7 shows $C D F(k)$ in carol and dnsjava when $\operatorname{sim}_{t h}$ is $0.1,0.3$, and 0.5 . $C D F(k)$ of 0.3 and $C D F(k)$ of 0.5 are not much different. $C D F(k)$ of 0.1 shows a coarse-grained distribution because $\operatorname{sim}_{t h} 0.1$ reduces the total number of genealogies. Figure 7 shows that our choice of $\operatorname{sim}_{t h} 0.3$ generates a finer-grained distri-
Table 4: The Average Size and Length of Genealogies with Varying sim $_{t h}$

\begin{tabular}{|c|c|c|c|c|}
\hline & \multicolumn{3}{|c|}{$\operatorname{sim}_{t h}$} \\
\hline & & 0.1 & 0.3 & 0.5 \\
\hline \multirow{2}{*}{$\begin{array}{l}\# \text { of genealogies } \\
\text { including false positives }\end{array}$} & carol & 27 & 122 & 153 \\
\hline & dnsjava & 63 & 140 & 180 \\
\hline \multirow{2}{*}{$\begin{array}{l}\# \text { of consistently } \\
\text { changed genealogies }\end{array}$} & carol & 16 & 41 & 53 \\
\hline & dnsjava & 21 & 45 & 52 \\
\hline \multirow{2}{*}{$\begin{array}{l}\text { avg size } \\
\text { (in \# of clone groups) }\end{array}$} & carol & 117.52 & 26.01 & 20.74 \\
\hline & dnsjava & 233.73 & 105.17 & 81.81 \\
\hline \multirow{2}{*}{$\begin{array}{l}\text { avg age } \\
\text { (length) }\end{array}$} & carol & 25.19 & 12.57 & 12.56 \\
\hline & dnsjava & 63.49 & 46.16 & 40.93 \\
\hline
\end{tabular}

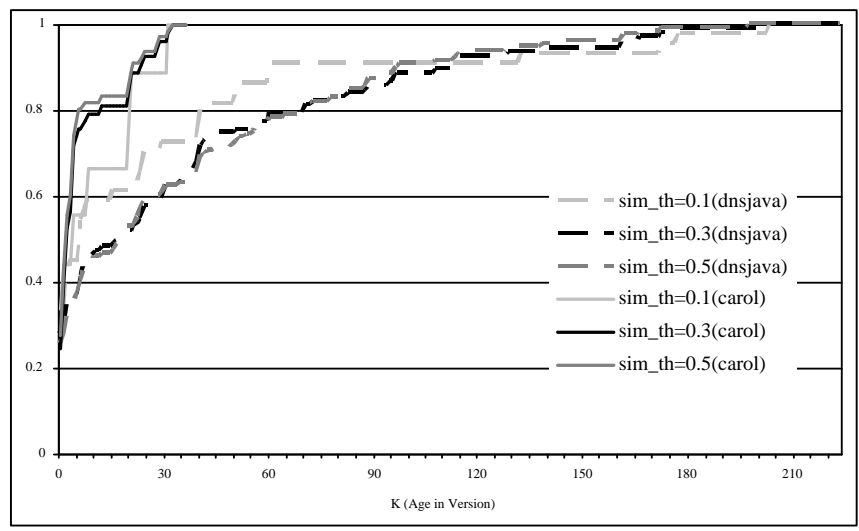

Figure 7: Cumulative distribution function of dead genealogies with varying $\operatorname{sim}_{t h}$

bution than using 0.1 and estimates the number of volatile genealogies more conservatively than using 0.5 .

\subsection{Study Limitations}

Clone Detection Technique. CGE incorrectly counts the number of consistent change patterns in some cases because CCFinder detects only a contiguous token string as a clone. ${ }^{5}$ For example, when code is inserted in the middle of one clone in a clone group, the existing clone group is broken into two new clone groups with shorter contiguous text; thus the change pattern would be identified as two consistent patterns rather than one inconsistent change pattern. As another example, even if a programmer consistently modified $O G$ to create $N G$, CCFinder does not find a cloning relationship between $O G$ and $N G$ if they do not share a contiguous token string greater than the size of $\operatorname{sim}_{t h}(|O G . t e x t|+\mid N G$.text $\mid) / 2$. In this case, the absence of a cloning relationship can be interpreted as a discontinuation of a lineage in our analysis. We speculate that this limitation can be overcome by plugging in clone detectors that find non-contiguous code clones, such as CP-Miner [26], PDGbased detectors [24, 22], and metric-based detectors [18, 27, $28]$.

Location Tracking Technique. We implemented a file and line based location tracker based on the diff algorithm; thus our location tracking algorithm is limited in two ways.

\footnotetext{
${ }^{5}$ Gemini, a clone analysis and visualization tool, identifies gapped clones (i.e., non-contiguous code clones) by postprocessing CCFinder's output [33].
} 
First, it depends on diff to resolve ambiguity in finding a corresponding line. For example, when a file $A$ contains $a b c$ in the $k-1^{t h}$ version and contains $c b a$ in the $k^{t h}$ version, diff considers that $a b$ is deleted before $c$ and $b a$ is inserted after $c$, even if a programmer replaced $a$ to $c$ and $c$ to $a$. Second, our algorithm considers that two files are not related when their file names do not match. For example, when a file $A$ is renamed to $B$ or $A$ is split into two files $B 1$ and $B 2$, the evolution patterns between $A$ and $B$ or $A$ and $B 1(B 2)$ would be identified as the Add and Subtract patterns. We speculate that code entity tracing techniques in Section 2 can improve our location tracker by inferring how classes were renamed, split, or merged.

Subject Programs. Our two subject programs both comprise about 20,000 lines of code. The clone coverage ratio of these programs was smaller than many programs reported in the literature. We speculate that carol and dnsjava may have fewer locally unfactorable and consistently changing clones than larger programs whose duplication is difficult to remove without compromising many existing design decisions. Both carol and dnsjava have been maintained by a small number of people: two developers for dnsjava and six developers for carol. The small team size may have affected our study results.

The granularity of our analysis was a check-in that changed the total number of lines of code clones (LOCC); thus we could not observe the changes between each check-in or the changes that did not result in $\triangle L O C C \neq 0$ even if the clones' text changed. Based on our experience of observing programmers copy and paste, we suspect that programmers create more clones temporarily before finding an appropriate level of an abstraction.

Our definition of locally unfactorable clones is Java language dependent; thus our claims about the locally unfactorable clones may not apply to other languages. We speculate that, in other programming languages, different types of locally unfactorable clones would be found depending on the language constructs.

\section{POTENTIAL TOOLS}

Our study results indicate that the problems of code clones are not so black and white as previous research has assumed and that there are several types of clones that refactoring would not help. We propose to use clone genealogy information to identify clones that may benefit from new clone maintenance approaches. Previously, we proposed several clone maintenance tools [21]. We discuss which types of clones the proposed tools target.

Suggesting When to Refactor. For clones that can be refactored, we believe that there may be an optimal time to refactor them. If programmers refactor code clones too early, they might not get the best return on their investment because the code clones may diverge or disappear. On the other hand, if programmers wait too long before they restructure code, they would get only marginal benefit on their investment. We envision that clone genealogy information, particularly the age of clones, could assist programmers in deciding when to refactor clones by differentiating volatile clones from long-lived clones.

Simultaneous Text Editing. Our result indicates that the longer clones live, the more they represent locally unfactorable and consistently changing clones. Refactoring techniques would not help maintain this type of clones con- sistently. Thus, we propose simultaneous editing of locally unfactorable and consistently changing clones. Simultaneous text editing is proposed for automating repetitive text editing and prototyped by Miller and Myers [29]. After describing a set of regions to edit, a user can edit any one record and see equivalent edits applied simultaneously to all other records. A similar editing technique, called linked editing, applies the same editing change to a set of code clones specified by a user [32]. These editing techniques require a user to manually specify what must be edited simultaneously. We envision that clone genealogy information can be used to automatically (or semi-automatically) select clones that would benefit most from simultaneous editing or linked editing.

Furthermore, we believe that many software engineering tools could leverage a rich set of evolution patterns in our clone genealogy model. For example, information about clones that have changed consistently for a long time but then change inconsistently later could be used to automatically place concerns to watch for in a bug database. As another example, information about the propagation of a copied snippet through a codebase could provide visual links in an integrated software development environment.

\section{CONCLUSIONS}

There has been a broad assumption that code clones are inherently bad and refactoring would remove the problems of clones. Thus, previous research efforts focused on mainly two areas: automatically detecting code clones and educating programmers on how to remove or avoid clones. To investigate the validity of this assumption, we built a clone genealogy extractor and investigated clone evolution structurally and semantically rather than quantitatively.

Our study of clone genealogy contradicts some conventional wisdom about code clones: (1) aggressive, immediate refactoring may not be necessary for many volatile clones, and (2) refactoring techniques cannot assist in removing many long-lived, consistently changing clones. We conclude that there are classes of clones that require different types of maintenance support than conventional refactoring. We propose that clone genealogy information can be used to identify clones that may benefit from new approaches to clone management.

\section{ACKNOWLEDGMENTS}

We thank Software Engineering Laboratory at the Osaka University for providing CCFinder and GRASE lab at the University of California, Santa Cruz for providing Kenyon. We also thank Annie Ying, Andreas Zeller, and anonymous reviewers for their detailed comments on our draft and Robert DeLine, Dan Grossman, Philippe Kruchten, and Tessa Lau for discussions that helped us refine our ideas.

\section{REFERENCES}

[1] http://www.cs.washington.edu/homes/miryung/cge.

[2] http://www.graphviz.org.

[3] Micromodels of Software: Lightweight Modelling and Analysis with Alloy. http://alloy.mit.edu, 2004.

[4] G. Antoniol, M. D. Penta, and E. Merlo. An automatic approach to identify class evolution discontinuities. In Proc. of the Int'l Workshop on Principles of Software Evolution, pages 31-40, 2004. 
[5] G. Antoniol, U. Villano, E. Merlo, and M. D. Penta. Analyzing cloning evolution in the Linux kernel. Information $\&$ Software Technology, 44(13):755-765, 2002.

[6] B. S. Baker. A program for identifying duplicated code. Computing Science and Statistics, 24:49-57, 1992.

[7] M. Balazinska, E. Merlo, M. Dagenais, B. Laguë, and K. Kontogiannis. Measuring clone based reengineering opportunities. In Proc. of the Int'l Software Metrics Symposium, pages 292-303, 1999.

[8] M. Balazinska, E. Merlo, M. Dagenais, B. Laguë, and K. Kontogiannis. Partial redesign of Java software systems based on clone analysis. In Proc. of the Working Conference on Reverse Engineering, pages 326-336, 1999.

[9] H. A. Basit, D. C. Rajapakse, and S. Jarzabek. Beyond templates: a study of clones in the STL and some general implications. In Proc. of the Int'l Conf. on Software Engineering, pages 451-459, 2005.

[10] I. D. Baxter, A. Yahin, L. M. de Moura, M. Sant'Anna, and L. Bier. Clone detection using abstract syntax trees. In Proc. of the Int'l Conf. on Software Maintenance, pages 368-377, 1998.

[11] K. Beck. extreme Programming explained, embrace change. Addison-Wesley, 2000.

[12] E. Burd and J. Bailey. Evaluating clone detection tools for use during preventative maintenance. In Proc. of the Int'l Workshop on Source Code Analysis and Manipulation, pages 36-43, 2002.

[13] S. Ducasse, M. Rieger, and S. Demeyer. A language independent approach for detecting duplicated code. In Proc. of the Int'l Conf. on Software Maintenance, pages 109-118, 1999.

[14] M. Fowler. Refactoring: Improving the Design of Existing Code. Addison-Wesley, 2000.

[15] M. Godfrey and Q. Tu. Tracking structural evolution using origin analysis. In Proc. of the Int'l Workshop on Principles of Software Evolution, pages 117-119, 2002.

[16] GRASE-Lab. User Manual: Kenyon. http://dforge.cse.ucsc.edu/projects/kenyon, 2005.

[17] Y. Higo, T. Kamiya, S. Kusumoto, and K. Inoue. Refactoring support based on code clone analysis. In Proc. of the Product Focused Software Process Improvement Int'l Conference, pages 220-233, 2004.

[18] J. H. Johnson. Identifying redundancy in source code using fingerprints. In Proc. of the Conference of the Centre for Advanced Studies on Collaborative Research, pages 171-183. IBM Press, 1993.

[19] T. Kamiya, S. Kusumoto, and K. Inoue. CCFinder: A multilinguistic token-based code clone detection system for large scale source code. IEEE Trans. Software Eng., 28(7):654-670, 2002.

[20] M. Kim, L. Bergman, T. A. Lau, and D. Notkin. An ethnographic study of copy and paste programming practices in OOPL. In Proc. of the Int'l Symposium on Empirical Software Engineering, pages 83-92, 2004.

[21] M. Kim and D. Notkin. Using a clone genealogy extractor for understanding and supporting evolution of code clones. In Proc. of the Workshop on Mining Software Repositories, pages 17-23, 2005.
[22] R. Komondoor and S. Horwitz. Using slicing to identify duplication in source code. In $S A S$, pages 40-56, 2001.

[23] R. Komondoor and S. Horwitz. Effective, automatic procedure extraction. In Proc. of the Int'l Workshop on Program Comprehension, pages 33-43, 2003.

[24] J. Krinke. Identifying similar code with program dependence graphs. In Proc. of the Working Conference on Reverse Engineering, pages 301-309, 2001.

[25] B. Laguë, D. Proulx, J. Mayrand, E. Merlo, and J. P. Hudepohl. Assessing the benefits of incorporating function clone detection in a development process. In Proc. of the Int'l Conf. on Software Maintenance, pages 314-321, 1997.

[26] Z. Li, S. Lu, S. Myagmar, and Y. Zhou. CP-Miner: A tool for finding copy-paste and related bugs in operating system code. In Proc. of Operating System Design and Implementation, pages 289-302, 2004.

[27] J. Mayrand, C. Leblanc, and E. Merlo. Experiment on the automatic detection of function clones in a software system using metrics. In Proc. of the Int'l Conf. on Software Maintenance, page 244, 1996.

[28] E. Merlo, G. Antoniol, M. D. Penta, and V. F. Rollo. Linear complexity object-oriented similarity for clone detection and software evolution analyses. In Proc. of the Int'l Conf. on Software Maintenance, pages 412-416, 2004.

[29] R. C. Miller and B. A. Myers. Interactive simultaneous editing of multiple text regions. In USENIX Annual Technical Conference, General Track, pages 161-174, 2001.

[30] E. Nickell and I. Smith. Extreme programming and software clones. In Workshop on Software Clones, 2003.

[31] K. Sullivan, P. Chalasani, S. Jha, and V. Sazawal. Software Design as an Investment Activity: A Real Options Perspective in Real Options and Business Strategy: Applications to Decision Making. Risk Books, November 1999.

[32] M. Toomim, A. Begel, and S. L. Graham. Managing duplicated code with linked editing. In Proc. of the Int'l Symposium on Visual Languages and Human-Centric Computing, pages 173-180, 2004.

[33] Y. Ueda, T. Kamiya, S. Kusumoto, and K. Inoue. On detection of gapped code clones using gap locations. In Proc. of the Asia-Pacific Software Engineering Conference, pages 327-336, 2002.

[34] T. Zimmermann and P. Weißgerber. Preprocessing CVS data for fine-grained analysis. In Proc. of the Workshop on Mining Software Repositories, pages 2-6, 2004.

[35] L. Zou and M. W. Godfrey. Detecting merging and splitting using origin analysis. In Proc. of the Working Conference on Reverse Engineering, pages 146-154, 2003. 